\title{
L'inachevé dans la modernité : littérature, art et musique
}

Adina Balint-Babos, Université de Winnipeg et Antonio Viselli, Université Trent

Qu'appelle-t-on une œuvre inachevée ? Qu'est-ce que finir un roman, terminer une toile, achever une partition ou une statue ? Quel sens a l'abandon d'une œuvre ? Dans sa fascination pour le tout et la beauté accomplie, l'esthétique classique refuse de poser ces questions et impose le silence sur les œuvres « imparfaites » d'un Michel-Ange ou d'un David, par exemple. Face à cette censure, l'époque moderne et contemporaine réclame l'inachevé, qui n'est plus accidentel mais passion des formes tâtonnantes, itinérantes, mouvantes, toujours en constitution. Avec Nietzsche, Proust, Joyce, les avant-gardes, Barthes, Duras et tant d'autres, l'inachevé et le fragment triomphent des pensées systématiques et témoignent de l'impérissable désir de représenter la vie qui se renouvelle incessamment.

On sait les attraits de l'inachevé : l'accident, l'abandon ou la mort donneraient à l'œuvre un supplément existentiel, au lecteur et spectateur le libre jeu esthétique des possibles, l'occasion d'un vagabondage, d'une flânerie. D'où le goût des ébauches, des esquisses, des projets interrompus. Johann Sebastian Bach fait paradoxalement du non-finito de la fugue un aboutissement, une visée. Sur un mode différent, le moderne work in progress légitime l'inachevé comme circonstance du processus de création et condition de «l'œuvre ouverte » (Eco 9).

Après tout, sur fond de quelle norme de l'achevé se définit l'inachevé ? Qu'est-ce que l'inachèvement d'un effet recherché d'inachèvement? Selon les contextes, il y a diverses manières d'interroger l'inachèvement d'une œuvre par rapport à une intégrité envisageable et envisagée. Sauf à prôner l'inachevé comme but, sa valorisation esthétique est, par exemple, liée à une prise de liberté par rapport à des contraintes et des codes, ainsi qu'au jeu et au plaisir que cette transgression entraîne. Par ailleurs, que l'œuvre soit entière ou non, achevable ou inachevable, et quels que soient les termes ou les critères variables de ce rapport à l'achèvement, ne convient-il pas d'envisager l'inachèvement, non pas comme un état, mais dans sa dimension active : comme un processus à l'œuvre?

La question centrale de notre dossier n'est pas l'inachevé comme incomplétude matérielle constatée et définitive, l'inachèvement passif d'une œuvre abandonnée avant l'accomplissement du dessein original. Les études réunies ici s'intéressent plutôt aux questions littéraires, poiétiques, 
esthétiques que suscite l'hypothèse d'un inachèvement actif, constitutif du geste de création en mouvement ou propre au projet. Si l'inachèvement passif nous oriente plutôt vers la réception, l'hypothèse d'un inachèvement actif remonte vers les poïétiques de l'inachevé à l'œuvre. La question n'est plus «qu'est-ce qu'une œuvre inachevée ? » mais «qu'est-ce qu'inachever une œuvre ? ». Cela nous conduit à des questions auxiliaires sur la notion même d'œuvre littéraire et d'œuvre d'art, sur le rapport de l'œuvre à l'idée d'un projet initial et d'une visée ultime, ou bien encore, sur l'improvisation.

L'inachevé semble nous renvoyer à une définition transgressive de l'art, au sens où l'art entend explorer l'au-delà des limites d'une règle ou d'une norme : limites classiques de la beauté et de la perfection; limites du sens et de la signification ; limites du médium, dont l'artiste s'efforcerait continûment de s'affranchir. L'hypothèse de l'inachèvement pose également la question du rapport de l'art au changement et à sa durabilité sous la forme d'un inachèvement actif, d'une œuvre toujours « rouverte », sans cesse en transformation, transformée et transformante, pour autant que selon Paul Valéry, «Euvre = transformer quelque chose en vue de transformer quelqu'un » (1404).

Entre mot, sujet et chose, il y a du jeu. Se situant dans cet écart, le poème contemporain ne peut être qu' « objeu », pour reprendre le terme de Francis Ponge - expression toujours provisoire et susceptible d'être remise en jeu. Il est connu que Ponge a pris de plus en plus délibérément, mais toujours avec humour, son parti de cet inachèvement perpétuel. À l'idéal du texte clos sur luimême, qui présidait au Parti pris des choses, il a substitué le «proême », poème en quête de luimême, qui associe à son élaboration sa propre poétique. Puis, rappelons le «carnet » et les brouillons eux-mêmes, publiés tels quels dans leur intégralité, en un geste qui confère au processus de composition du texte une dignité égale à celle de son état ultime. Ponge rejoint ainsi une tendance de l'art moderne, qui valorise le work in progress.

Une question surgit ici en filigrane : existe-t-il des œuvres que l'on puisse dire parfaitement achevées ? Comment comprendre qu'un texte littéraire puisse donner lieu à la fois à des commentaires, eux-mêmes infinis, et à des récritures ? Dans « La critique et l'invention », Michel Butor nous invite à ne pas séparer les deux phénomènes en posant que la postérité d'une œuvre, dans les interprétations dont elle est l'objet, comme dans les textes seconds qu'elle engendre, relève d'un procès de continuation. De fait, que nous transmet le destin d'une œuvre, dans la 
pluralité des interprétations auxquelles elle a pu donner lieu, sinon qu'elle reste de quelque façon « inachevée »?

À un autre niveau: insatisfaction permanente de l'écrivain face à l'œuvre écrite, l'achèvement serait-il en faveur d'une œuvre nouvelle qui requiert de lui qu'il se détache du travail passé en le livrant au public ? Didier Anzieu le souligne dans son ouvrage sur le « corps de l'œuvre ». Songeons un instant au Cimetière marin de Valéry qui n'a vu le jour que par l'intervention de Paulhan qui arracha le manuscrit au poète - lequel ne parvenait pas à voir son œuvre comme achevée ; Marcel Proust qui remania jusqu'à la fin de sa vie l'architecture de Sodome et Gomorrhe sans parvenir à fixer définitivement la place d'Albertine disparue, ou encore à L'arrêt de mort de Maurice Blanchot dont l'auteur a conçu deux versions successives (1948 et 1972), sans signaler au public les variantes introduites ${ }^{1}$. Au dix-neuvième siècle, Le chef d'œuvre inconnu d'Honoré de Balzac est peut-être la plus belle allégorie de la tâche infinie qui est celle de l'artiste. Une question s'impose naturellement : quand donc un poète, un écrivain ou un artiste peut-il dire son œuvre parfaitement achevée?

À la suite de Philippe Lacoue-Labarthe et Jean-Luc Nancy dans L'absolu littéraire. Théorie de la littérature du romantisme allemand (1978), il est évident que l'œuvre moderne ne fait que radicaliser un trait constitutif de toute œuvre littéraire : tout se passe comme si, depuis le Romantisme allemand qui a théorisé le fragment, les écrivains de la modernité écrivaient dans la conscience de l'inachèvement essentiel des œuvres littéraires. Pourtant, on peut supposer que cette conscience s'est forgée à la lecture des œuvres du passé qui manifestent cet inachèvement, œuvres « ouvertes » avant la lettre, car proprement infinies ou restées inachevées. Rappelons les Essais de Montaigne ou les Pensées de Pascal, qui sont à l'évidence des modèles pour Butor dans son effort de définir l'œuvre «moderne $»^{2}$. D'un point de vue théorique, l'hypothèse d'un inachèvement constitutif des œuvres littéraires présente des avantages intéressants - elle permet d'unifier le triple processus qui régit la constitution d'un texte : le processus poḯtique (la genèse d'une œuvre), le processus rhétorique (la dynamique de sa lecture) et le processus herméneutique (les interprétations auxquelles le texte a pu donner lieu). Ainsi, on est conduit à envisager dans les termes d'une continuation ou de l'invention de variantes, la production du texte, sa réception par le lecteur et son devenir historique, en posant des liens entre les trois formes majeures de transtextualité : le métatexte, l'intertexte et l'hypertexte (Genette 10). 
Les contributeurs du dossier montrent une diversité d'analyses et d'approches méthodologiques autour de la question de l'inachevé. D'une part, les articles interrogent les notions d'infini, d'intermédialité et de créativité, ainsi que des formes d'expression de la mémoire inachevée d'Auschwitz, du deuil et de la mélancolie. La pensée philosophique de Nancy sur la notion de «de trop», les réflexions de Roland Barthes sur le fragment et les concepts d'anachronisme et de montage de Georges Didi-Huberman servent de support théorique à déchiffrer les facettes de l'inachèvement dans la littérature, les arts visuels et la musique dans la modernité.

D'autre part, les auteurs des études critiques explorent l'inachèvement et sa mise en œuvre dans des analyses de textes appartenant à un corpus varié du vingtième siècle jusqu'à l'époque contemporaine : Gide, Perec, Barthes, Cendo, Didi-Huberman, ce qui nous conduit à réfléchir aux rapports entre le visuel, le scriptural et le musical dans les études littéraires et l'histoire de l'art et des idées. Après tout, comment comprendre les multiples représentations de l'inachevé dans différents médiums de création?

Les articles présentés ici approfondissent la réflexion sur l'inachevé comme phénomène inhérent à la création d'une œuvre et comme possibilité d'interprétation de cette œuvre. Ainsi, Cosmin Toma examine le dispositif inachevé d'Introduction aux ténèbres, partition signée Raphaël Cendo et interprétée par l'ensemble de musique contemporaine Ictus, dirigé par GeorgesÉlie Octors à Strasbourg en septembre 2010. Dans son analyse, Toma convoque « l'art des sons » de Nancy. Pour sa part, Daniele Carluccio étudie la poétique du deuil de l'achèvement dans le $W$ ou le souvenir d'enfance de Georges Perec, en montrant que « $W$ est bien articulé autour d'une place vide, qui est montrée, mais celle-ci n'est pas à remplir, elle attire plutôt le tout dans sa béance », ce qui justifie, selon l'auteur, le ton mélancolique du récit. Quant aux écrits de la Shoah, Adina Balint-Babos explore l'inachèvement à partir d'Écorces, le récit-photo de Didi-Huberman. Elle souligne que les images des camps de concentration nous reviennent comme des images documentaires rares : ces images-là sont « des images-symptômes, elles se déplacent, flottent », et nous incitent à réfléchir sur la connaissance d'un événement tragique de l'histoire par le montage du texte et de l'image.

Dans un article qui prend la forme d'un dialogue entre la littérature et la musique, Antonio Viselli analyse Les faux-monnayeurs d'André Gide comme ouvrage marqué par l'inachevable, susceptible d'entrer en résonnance avec L'art de la fugue de Bach. En se penchant sur la notion de 
discours polyphonique chez Mikhaïl Bakhtine, Viselli met en lumière les croisements de la fugue littéraire et de la fugue musicale, ce qui rappelle «le théorème de l'incomplétude du mathématicien Kurt Gödel [qui] évoque l'élan interminable de la forme fugale en musique et en littérature ». Pour terminer, Elizabeth Geary Keohane étudie le thème de l'inachèvement dans les Carnets d'Egypte de Gide. En faisant appel à l'ouvrage inachevé d'Edward Said, On Late Style, Geary Keohane situe les Carnets de Gide dans un contexte plus large, celui du rapport entre la créativité, le spectre de la mort et les aléas du processus de création.

Finalement, si la littérature, l'art et la musique nous conduisent à aborder la question de l'inachèvement à partir du processus de création, des interprétations de textes et des textes euxmêmes, il s'agit à la fois de réfléchir à l'inachevé comme médium qui permet de jeter des passerelles entre les arts, les cultures et les productions esthétiques. En même temps, cela nous invite à penser la question de l'inachèvement comme une question transculturelle, étroitement liée aux déplacements des populations et aux transferts culturels qui caractérisent l'époque contemporaine. Car, « [d]e nos jours, tout se déplace, tout se recontextualise $[\ldots]$ par la capacité à pénétrer les réseaux mondiaux de savoirs » (Imbert 10), ce qui signifie certes qu'on assiste à une rencontre des poétiques, des imaginaires, à une mise en réseaux des discours, des pratiques artistiques et des moyens d'expression, à un métissage des formes et des genres favorables à l'inachevé.

\section{Bibliographie}

Anzieu, Didier. Le corps de l'œuvre. Essais psychanalytiques sur le travail créateur. Paris: Gallimard, 1981.

Balzac, Honoré de. Le chef d'œuvre inconnu. Paris : Livre de poche, 1995.

Blanchot, Maurice. L'arrêt de mort. Paris : Gallimard, 1977.

Butor, Michel. «La critique et l'invention». Répertoire III. Paris : Minuit, 1968. 111-113.

Eco, Umberto. L'œuvre ouverte. Paris : Seuil, 1979.

Escola, Marc. Brèves questions d'herméneutique. Paris : Champion, 2001.

Genette, Gérard. Figures III. Paris : Seuil, 1972.

Imbert, Patrick. Le transculturel et les littératures des Amériques. Ottawa : Chaire de recherche de l'Université d'Ottawa: Canada: enjeux sociaux et culturels dans une société du savoir, 2012. 
Lacoue-Labarthe, Philippe et Jean-Luc Nancy. L'absolu littéraire. Théorie de la littérature du romantisme allemand. Paris : Seuil, 1978.

Madaule, Pierre. Une tâche sérieuse ? Paris : Gallimard, 1973.

Ponge, Francis. Le Parti pris des choses. Paris : Gallimard, 1942.

Proust, Marcel. À la recherche du temps perdu, tome II. Paris : Gallimard, Pléiade, 1988.

Valéry, Paul. « Notion générale de l'art». Euvres, tome I. Paris : Gallimard, Pléiade, 1957.

\section{NOTES}

${ }^{1}$ Voir Pierre Madaule.

${ }^{2}$ Voir Marc Escola, chapitre X : « Ce que peut un fragment ». 